\title{
Construction of Water Surface Topography Using LIDAR Data
}

\author{
Gašper Rak ${ }^{1, *}$ - Marko Hočevar ${ }^{2}$ - Franci Steinman ${ }^{1}$ \\ ${ }^{1}$ University of Ljubljana, Faculty of Civil and Geodetic Engineering, Slovenia \\ 2 University of Ljubljana, Faculty of Mechanical Engineering, Slovenia
}

\begin{abstract}
Measurements of water surface topography are important for hydraulic structures, operation of hydropower plants as well as in the determination of water surface profiles in rivers, especially in the event of high waters. We therefore investigated the conditions at a confluence of two supercritical flows, where distinctly three-dimensional flow conditions of standing waves form, as well as an unsteady structure of the water flow in transversal and longitudinal directions. Due to the fast water surface dynamics and the phenomenon of foamed or twophase flow, the conventional measurement methods typically used in hydro engineering are not suitable for capturing complex water surface topography with high temporal and spatial resolution. Hence we wanted to verify the appropriateness of the laser scanning method for water surface topography measurements. This measurement method, which is considered less suitable or even useless for measurements of water body surfaces, was, coupled with an innovative approach, successfully used for water surface measurements of dynamic, turbulent, two-phase water flow. The acquisition of a point cloud with high temporal and spatial resolution allows for the construction of topography of intensive waving, which will also enable a topology analysis based on a phenomenological analysis of the relations between integral parameters of water flows and standing wave characteristics at the confluence.
\end{abstract}

Keywords: laser scanner, water surface, topography, two-phase flow, confluence

Highlights

- $\quad$ Non-intrusive measurements of highly aerated, turbulent water flow was performed.

- Laser scanning enables acquisition of free-water surface profiles with high spatial and temporal resolution.

- $\quad$ The vertical fluctuations of non-stationary water surface were defined with post-processing of LIDAR data.

- The raw point cloud of laser scanning was used to construct water surface topography.

\section{O INTRODUCTION}

Measurements of free surface flows are important in a wide range of water engineering application in order to understand these flows and validate predictive tools we need to measure the free surface elevation accurately. It is also important for mechanical engineering, since water surface fluctuations affect the boundary conditions of mechanical equipment (e.g. at Hydropower plant (HHP)). Two hydraulic phenomena showing the significance of measuring free-water surface profiles are well illustrated in Fig. 1. In order to determine flow conditions, it is also necessary to measure such water surface dynamics as found during high flows below the hydropower plant (HPP) (Fig. 1a), as well as in different laboratory experiments, where turbulent, two-phase flow is present. Therefore, an experimental apparatus was set up at the hydraulic laboratory of the University of Ljubljana, Faculty of Civil and Geodetic Engineering, with high dynamics and diverse distribution of the water surface in a right-angled channel, which allowed us to test the applicability of laser scanning for aerated water surface measurements (Fig. 1b).
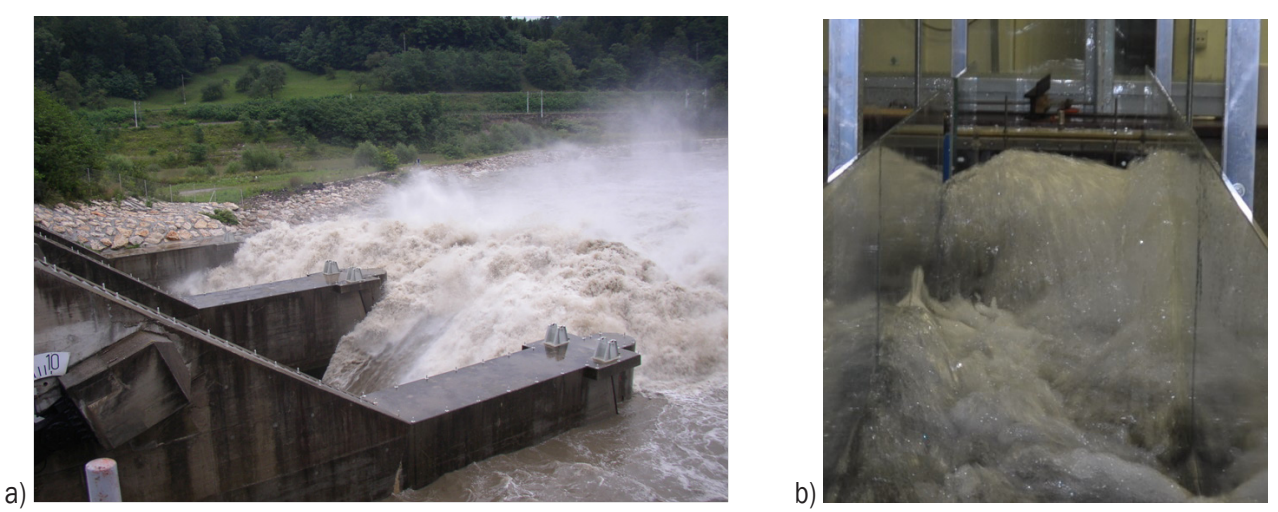

Fig. 1. a) The dynamic water surface below the Vrhovo HPP, and b) the experimental apparatus at the hydraulic laboratory, where distinctively standing waves are formed at the confluence of two incoming supercritical flows 
As an interesting practical case, a confluence of two flows was selected, where even with small velocities and with otherwise fairly calm water surface, vortexing and distinctively 3D flow conditions occur at the confluence. Insight into the situation at confluences has a wide applicability both for users and spatial planners, as confluences occur both on streams (natural and artificial river channels, torrents, etc.) as well as on many facilities and water infrastructure, such as fish passes, water treatment plants, surface water drainage from paved surfaces, etc. Knowledge of these phenomena is particularly important when designing the freeboard of longitudinal structures along the watercourse (e.g. bank fortifications along traffic routes) and transversal structures such as culverts and bridges that could be exposed to such waves.

\section{WATER SURFACE TOPOGRAPHY AT THE CONFLUENCE}

A marked change in flow conditions is caused by the confluence of two flows when, depending on the relationship between the magnitudes of the incoming flows, a more or less complex water surface topography. The conditions at confluences with incoming supercritical flow, with a high linear momentum, are even more complex, causing intensive transversal water mass dynamics. Similar to phenomena such as hydraulic jump, breaking waves etc., this is a distinctively three-dimensional water phenomenon, where a time-varying structure of the water flow forms in the transversal and longitudinal directions, which we are still unable to fully simulate using three-dimensional numerical models. 3D models are still not widely used in engineering work as they are too complex, while measured surface topographies allowing for calibration or verification of calculations are available only rarely. The acquired experimental data will be important for the development, calibration, and verification of 3D numerical models to obtain reliable results for the design and operation of hydraulic structures. In practice, particularly on torrential streams, this would help to prevent the under dimensioning of structures, thus no longer failing to take into account the accompanying processes deteriorating the functionality of hydraulic structures. Measurements are thus important, both in the field and in the laboratory, as they help us gain new insight into and knowledge of the phenomena and processes therein, while the applicability of measuring equipment can also be tested in the laboratory for more complex field conditions.
A detailed review of the literature shows that flow conditions at confluences are frequently studied, both experimentally and numerically; however, studies in subcritical flow regime prevail, i.e. where the water surface breaks into waves only slightly (e.g. [1] to [3]). Field measurements were also taken in subcritical flow conditions, which involved the triangulation of a reflector mounted on a small raft, which could only be done on a slightly undulating water surface at the confluence [4]. Few studies analysing flow conditions with supercritical flow across the confluence have been published (e.g. [5] and [6]), while the transversal changing of surface water formation and pulsations is studied the least. The main reason certainly lies in the unsuitability of conventional measurement methods, e.g. measurements of local pressures using piezometers, ultrasonic sensors, or point gauges. They measure flow parameters at a point, thus interfering with the water flow, or their performance is limited when two-phase flow occurs; thus, they do not allow for dynamic measurements with high spatial resolution. To successfully capture the dynamics of water surface topography, the measuring methods should allow for sampling using frequencies that are considerably higher than the changing rate of the water surface, it should have high spatial resolution, while the individual measuring equipment elements should not interfere with the flow and thus affect flow conditions. In our study we checked whether laser scanning could be used for these purposes. The measuring method used for a wide range of both professional and research fields to measure surfaces and/or terrain, was, until now, mostly considered as less suitable or successful in terms of measuring water body surfaces ([7] to [9]). Some studies show that laser scanning can be used to measure time-varying water surface formation ([10] to [13]), but only when particulate matter is already in water or it is added to improve reflectivity, which, however, reduces the usability in field measurements.

In analysing surface waves, along with average topography values, average values within very short time frames are important (i.e. pulsations), based on which the wave dynamics at a relevant travel length can be assessed. Our studies have shown that LIDAR can be used to measure water surfaces without the addition of particulate matter [14] in hydraulic phenomena that include turbulent twophase and foamed flow, i.e. particularly in cases when other measuring methods provide less reliable, often unsuited results. In this study, the advantages of laser scanning were shown in the case of a very complex water surface topography formed at confluences with 
supercritical flow. A literature review shows that due to the limitations of conventional measuring methods this kind of topography measurements have not been taken so far.

\section{EXPERIMENTAL APPARATUS AND MEASURING EQUIPMENT}

The studies, which were divided into two parts, were carried out at hydraulic laboratory. First, we had to find the appropriate measuring equipment and define the settings of its parameters (having in mind that it would be used in field conditions as well) and verify the suitability of LIDAR technology for measuring water surfaces. An extensive verification process was conducted of both standing water and turbulent twophase flow with high vertical water surface dynamics. Here, the verification procedure is not provided in detail as it relates to the measuring method, which is described elsewhere [14]. In the second part, systematic topography and surface pulsation measurements were taken at a right-angled confluence of two supercritical flows (hereinafter: T-junction, Fig. 2). The results of these extensive measurements were used to produce mesh models of water surface topography at T-junctions.

\subsection{Experimental Setup of a T-Junction}

The T-shaped junction, with a $90^{\circ}$ angle between the axis of the main channel and its side inflow, has a 6-m long main channel and a 1-m long side channel. The experimental apparatus was entirely made of glass, which reduced the effect of walls on flow conditions as well allowed for a side view of the flow conditions. A horizontal bottom of all sections was provided to optimise the measurements and particularly to allow for appropriate inflows to the model, to minimise the number of joints at the confluence that might bring additional disturbances, and to reach the desired water flow velocities at the channels. The coordinate system of experimental set up has its origin of longitudinal axis at the beginning of the main channel, the origin of transverse axis at the middle point of the main channel, and of the vertical axis at the bottom of the channel (Fig. 2a).

To achieve a more distinct dynamic at the confluence, the conditions during supercritical flow were analysed when along with the flow structure a more complex water surface topography is developed in the form of standing waves, whose height significantly exceeds the average water surface levels of incoming flows or the flow downstream the confluence after calming of the waves (Fig. 2b).

Fig. 3 shows the water surface dynamics at the confluence, when the side inflow pushes away the main flow towards the opposite wall, which is followed by a downstream left/right fluctuation of the water mass until the transversal movement settles down. To gain insight into the standing wave formation it is necessary to measure wave peaks and their location as well as the magnitude of transversal fluctuation.

The dynamics at the confluence depend on the relationship between the energy and the linear momentum of both inflows. Given the selected events, the desired inflows in the inlets and at the confluence itself were provided with a controlled inflow to the model through two pressure vessels with adjustable height of the openings (Fig. 4a), and thus the height of the incoming water. The height of the apertures was adjustable to an accuracy of $0.01 \mathrm{~mm}$. We can assume that due to the short distance between the outflow from the vessel and the start of mixing of the two flows at the confluence, and the small friction
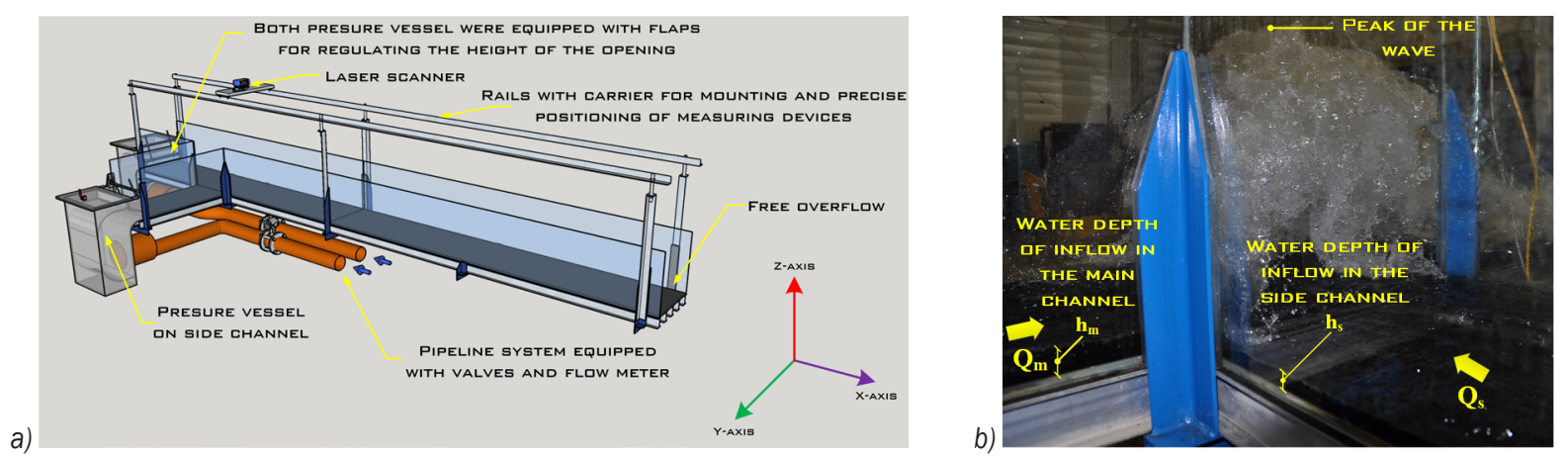

Fig. 2. a) A drawing of the right-angled confluence, and b) the photo of the waves at the confluence of supercritical flows exceeding the depth of the incoming flows by several times 

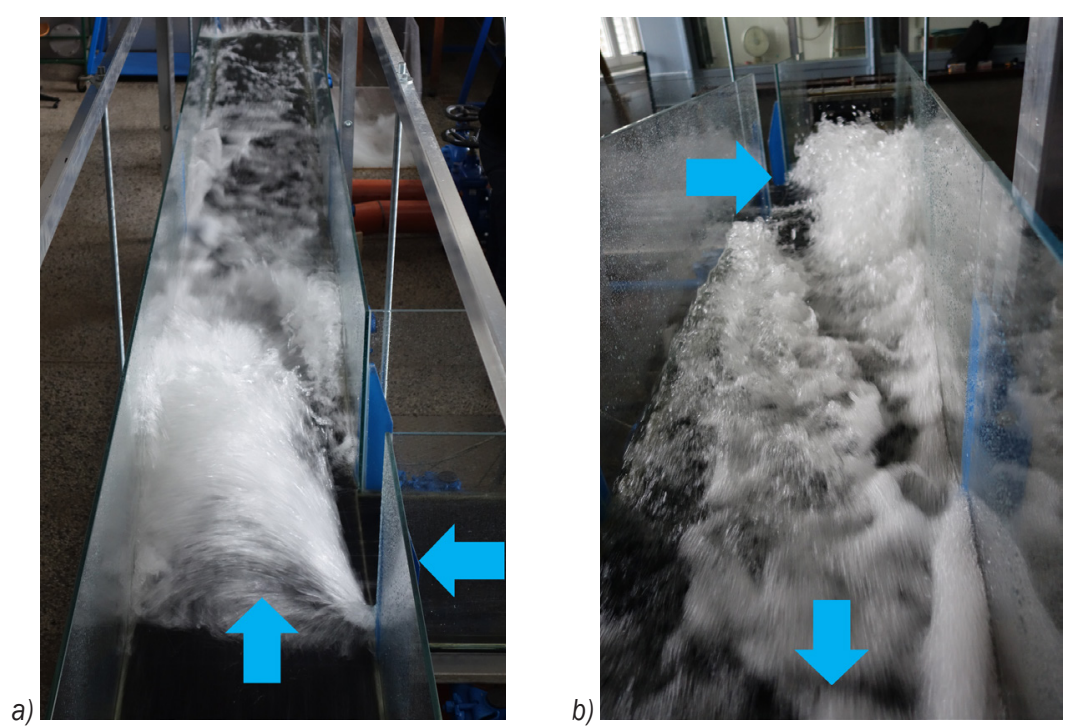

Fig. 3. Photos of the apparatus show the dynamics of flow conditions at the confluence of two incoming flows; a)downstream view, and b) upstream view)
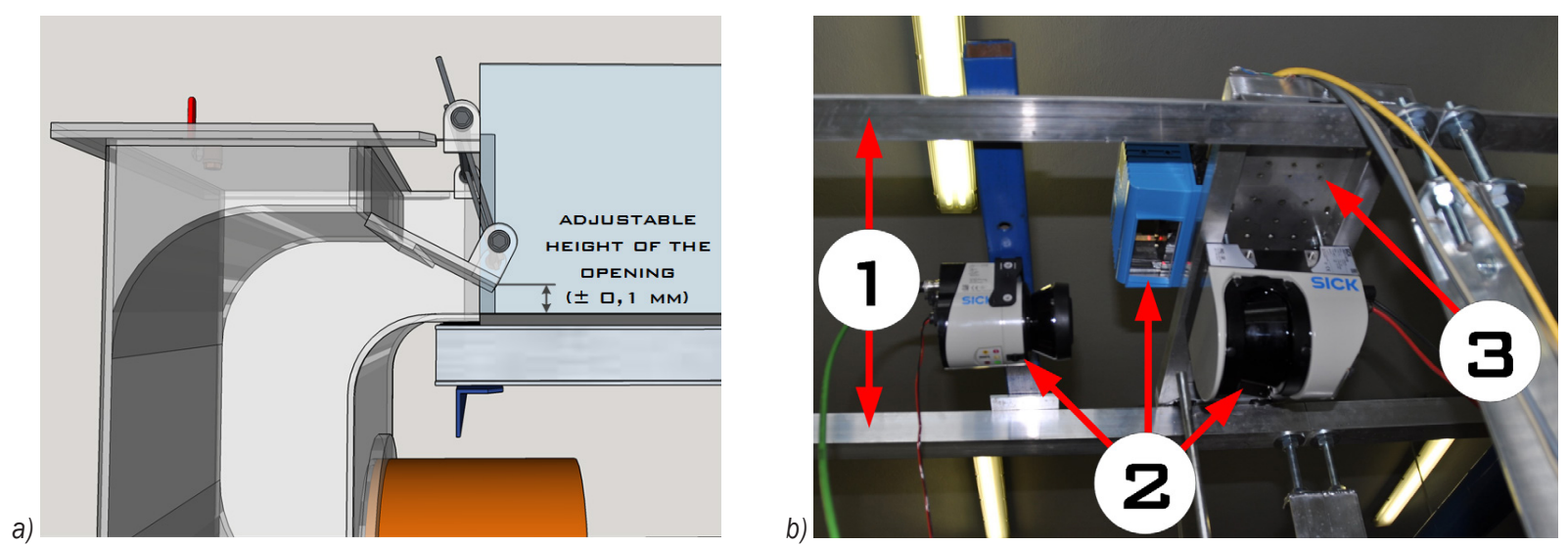

Fig. 4. a) The pressure vessel with a flap for regulating the height of the inflows; $b$ ) the frame structure (1) with the measuring equipment (2) mounted on a special longitudinal carrier (3) whose structure allows for precise positioning in measured cross-sections

at the glass walls the inflow height and depth were preserved in this section.

The experimental apparatus was equipped with a support provided by rails with a rail carrier for mounting measuring devices and a mechanism that allowed for repeatability of measurements in selected cross-sections with a precise location of the measuring equipment (Fig. 4b).

The frame structure for measuring equipment installation was separately attached to the model base, thus preventing the transmission of vibrations from the glass channel to the measuring equipment and the occurrence of additional pulsations and measurement uncertainty.

\subsection{Measuring Equipment and Its Characteristics}

LIDAR instrument LMS400 manufactured by SICK AG was used for water surface measurements. Based on the comparison of the measurements using different laser measuring systems this laser scanner proved to be the most suitable. Laser scanner operates in the visible red light wavelength $\lambda=650 \mathrm{~nm}$ (Fig. $5 a)$. It is used for both indoor and industrial use with distances not exceeding $7 \mathrm{~m}$. With this measuring range the measurement uncertainty is low also, i.e. $\pm 3 \mathrm{~mm}$ with solid bodies. The instrument allows for a selection of the combination of scanning frequency $270 \mathrm{~Hz}$ to $500 \mathrm{~Hz}$ and an angular resolution from $0.1^{\circ}$ to $1.0^{\circ}$. According to the manufacturer, the nominal accuracy of measurements can be achieved even at 


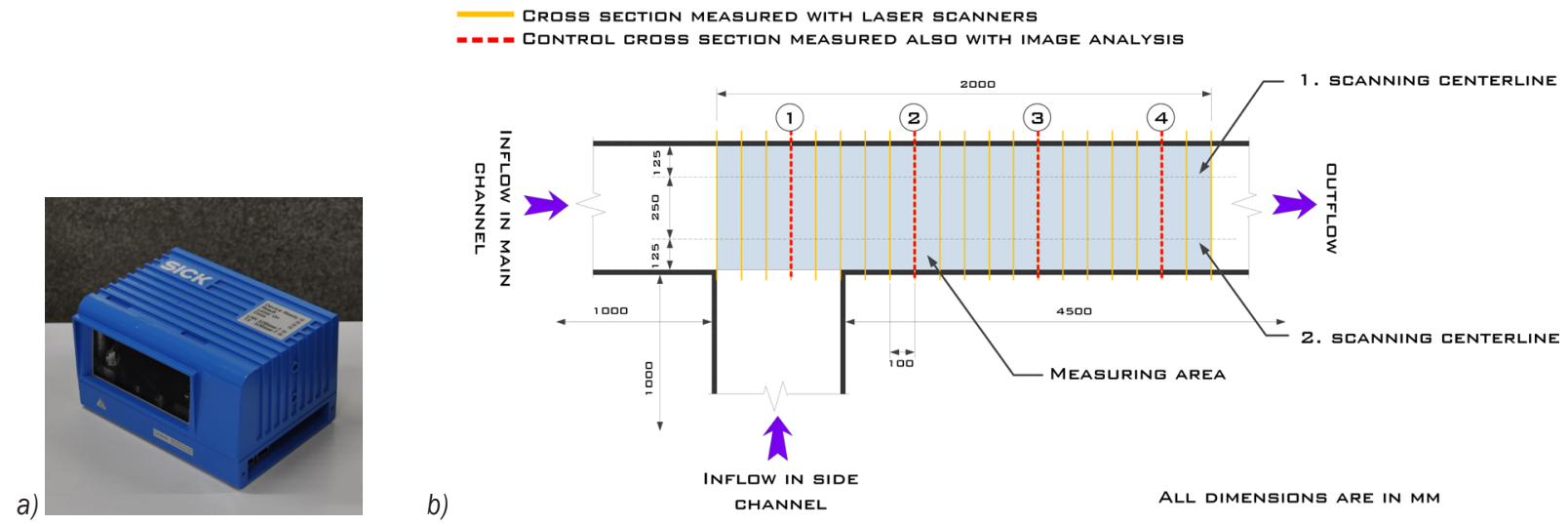

Fig. 5. a) Laser scanner SICK LMS400, and b) a scheme of the T-junction with its main dimensions, measured cross-sections, and the two lonsitudinal lines where simultaneous measurements in all cross-sections were taken

$290 \mathrm{~Hz}$ or at an angular resolution of $0.2^{\circ}$ or at 500 $\mathrm{Hz}$ with an angular resolution of $0.4^{\circ}$. For an optimum number of the measured reflections in the individual recordings of cross-sections we used a frequency of $269.8 \mathrm{~Hz}$ and an angular resolution of $0.2^{\circ}$. To eliminate systematic errors or reduce their influence, test field measurements at a precisely known distance were taken before each set of measurements.

The longitudinal rails ensured that in all cases the bottom of the channel was at a distance of 1150 $\mathrm{mm}$ from the scanner. The range of depth fluctuations was between $0 \mathrm{~mm}$ and $450 \mathrm{~mm}$ so distances from $1150 \mathrm{~mm}$ to $700 \mathrm{~mm}$ were measured, while all measurements were taken inside the measuring range of the scanner; due to the response time of the device it was important that the measured surface was not located too close to the device.

Verification of the Lidar measurements using conventional instrumentation was not possible due to the highly complex and aerated nature of the freewater surface. Therefore, the verification of the laser measuring method in the dynamic water surface with two-phase flow was conducted by determining the reference values through analysing a sequence of images. Water level fluctuations were recorded using a high-speed camera, while a thin metal ruler was placed in the water to measure absolute values; the influence of the ruler under the given flow conditions could be assumed as negligible. The water surface was illuminated with a laser beam right at the ruler to later ease image processing, and particularly to improve resolution. A laser delivering $5 \mathrm{~mW}$ of output power and a operational wavelength $650 \mathrm{~nm}$ was used for illumination. Even though the directional light was used for illumination of the water surface at the ruler, bright strip was still thick around $1 \mathrm{~cm}$ (due to foamed water and bubbly flow). Casio EX-F1 highspeed camera was used which allows for up to 300 images per second, while a frequency of 60 images per second, with a resolution of $1920 \times 1080$ pixels, would suffice for water surface recordings. Video recordings of a length of $10 \mathrm{~s}$ were processed using our own algorithm for determining the mean value and fluctuations in Matlab. The verification measurements were conducted in 4 control sections. They are marked with red line and numbered with 1 to 4 on the Fig. 5 . Verification of the method with image analysis was conducted with manual (visual) control of water levels determined with post-processing of video sequences.

To obtain the topography of standing waves at the confluence, measurements were taken in 20 cross-sections at intervals of $100 \mathrm{~mm}$ (Fig. 5b). For the individual scenarios of flow conditions a total of 40 cross-sections in two parallel longitudinal axes were recorded. At a higher incidence angle of the beams the number of received echoes is lower due to higher energy dissipation of the beams and specular reflections from the surface. The two scanning centerlines, as the $1^{\text {st }}$ and $2^{\text {nd }}$ centerlines in all measured cross-sections, are marked on Fig. 5b. This type of recording especially improved the quality of measurements along walls and in places where major changes in water surface topography occurred. In the case of a single centerline recording, such changes in topography could cause blind spots, failing to capture the entire cross-section.

At various inflow conditions, a total of 168 scenarios were recorded, where Froude numbers, which are used to express the intensity of supercritical flow, varied at both inflows between 2 and 12, while water depths varied between $10 \mathrm{~mm}$ and $30 \mathrm{~mm}$. 3D models of topography for 5 scenarios are presented 
later in the paper. Raw data were recorded as a $2 \mathrm{D}$ point cloud in the polar coordinate system both when verifying the measuring method (which is not described here) and during systematic scanning of cross-sections along the confluence. A transverse profile of water surface for each measured crosssection was determined from the point cloud, which was composed of 6000 scans. The number of the measured values in the individual scans of the measured surfaces was a product of the angular range of measurements and the angular resolution. At an angular range $70^{\circ}$ and an angular resolution of $0.2^{\circ}$, the number of outgoing signals is thus 350 per scan, i.e. a total of 2,100,000 points in the point cloud of a selected cross-section. Despite huge number of signals, emitted from laser scanner, only around $5 \%$ of reflected beams are detected by the LIDAR scanner, and around $30 \%$ of returned beams have remission values over threshold.

On the water surface of turbulent free-surface flows different types of two-phase air-water flow can occur (e.g. dispersed bubble flow, bubble flow, a foamed upper layer of water body and splashing of water droplets of different sizes). Because of that it is very difficult or even impossible to determine the water surface exactly. From that reason it is very important that a post-processing of LIDAR point cloud enables determination of the range of fluctuations. With filtering the measurements on the base of reflection intensity of the individual emitted signals, it is also possible to eliminate measurements, where returned beam was reflected from droplets and splashing.

\subsection{Processing of the Raw Point Cloud of Measurements}

In the first part of processing, the data on the measured distances were converted into the Cartesian coordinate system and point clouds from both positions of laser scanners were combined. Given the known channel geometry, the points representing the measurements outside the channel were excluded. By filtering the points based on the measured distances or calculated coordinates in the Cartesian coordinate system, the echoes received after several reflections from various surfaces were also excluded. In such a scenario, the laser scanner receiver receives the return signal, but due to the longer time of flight, the measured distance is too long, deviating from the calculated values of the coordinates within the area in question, i.e. the channel.

Along with the measured distances, reflection intensity for the individual emitted signals was also obtained, as based on the various intensities it was possible to exclude the measurements, i.e. signal returns from the drops above the water surface or from the bubbles deeper in the water body. Due to light scattering and energy dissipation along the path of the beam through water, these measurements revealed significantly lower remission values of signal returns. Such characteristics were analysed with the measurements of standing water, where, at various water depths, bubbles moving from the bottom to the surface were injected, thus determining the threshold that was later used in filtering laser measurement data [14]. The threshold was, subject to testing, set to a value that allowed for the exclusion of echoes from the bubbles deeper in the water and the smaller drops in the air, while still ensuring that most of the measured points were taken into account for calculating the water level's mean value [14]. The constant value of threshold was used for all measurements of water surface topography at the confluence.

Because that the returned beam was often not detected by the laser scanner or reflection intensity of returned beam was under threshold, data are randomly missing and consequently the data were nonuniformly sampled. Taking into account all successful measurements of each scan the water surface was determined with interpolation in uniformly increasing $\mathrm{Y}$ coordinates (transverse axis; increment of $5 \mathrm{~mm}$ ). Set of data obtained was used for determination of water surface profile in a selected cross-section using median function. In the next step interpolation for 2D gridded data (predefined coordinates in $\mathrm{X}$ and $\mathrm{Y}$ direction between measured profiles; in both direction was used increment of $5 \mathrm{~mm}$ ) was used to get data set to construct final 3D mesh models of water surface topography.

\section{RESULTS}

The measurement method verification, which first took place in a chamber with controlled conditions, showed that LIDAR can be only used to capture the formation of water surfaces where foamed or developed enough two-phase flow in the upper layer occurs. Due to the laser beam reflections from the water surface, successful measurements in standing, clean water were limited to an incidence angle of the beams at around $0^{\circ}$. In poorly developed two-phase flow with low bubble density and when bubbles occur across the entire depth of the water body, there are more reflections and a wider range of incidence angles. The assessed values are lower than the actual ones due to the reflections from the bubbles deeper in the water. 
The influence of the bubbles deeper in the water was successfully reduced by filtering the point cloud and taking into account the intensity. In the foaming upper layer of standing water a measurement accuracy of \pm 3 $\mathrm{mm}$ was achieved, which corresponds to the nominal measurement uncertainty of the LIDAR device [14].

The verification of the measurement method at the T-junction apparatus, made e.g. in the scenario involving strong vertical water surface dynamics (surface fluctuation up to $\pm 50 \mathrm{~mm}$ ) and the two-phase flow phenomenon, showed that due to the dynamic nature of the phenomenon and the measured medium as well as the estimated measurement uncertainty of the reference method, by analysing the images taken using the high-speed camera, the measurement accuracy can be estimated at $\pm(5$ to 10$) \mathrm{mm}$. The case of the measured transversal formation of the water surface and the comparison with the reference values determined by analysing the images taken with the high-speed camera for cross-section 2 (Fig. 5b) is shown on Fig. 6 and in Table 1. The point cloud, presented on the Fig. 6, consist of 3000 scans with the laser scanner, measured from a single location (above the middle of the channel). In measurements using image analysis water surface fluctuations were also detected shown with a deviation interval from the mean value.

Fig. 6 shows that the water surface profile, determined by laser measurements and filtering based on remission values, agrees well with the measurements taken using the high-speed camera. Minor differences are shown in Table 1.

Based on the data processing shown and the small deviations in measurements in control cross-sections (Fig. 5), LMS400 was selected. All topography measurement depict the most dynamic part of the water surface along the confluence, as it gives the most important information for design, operation, etc. The mean values of the measured surfaces in consecutive cross-sections were used to construct the dynamic surface area, as a measured water surface topography of the area concerned, and presented from surfaces in the form of 3D mesh models (Fig. 7). Only

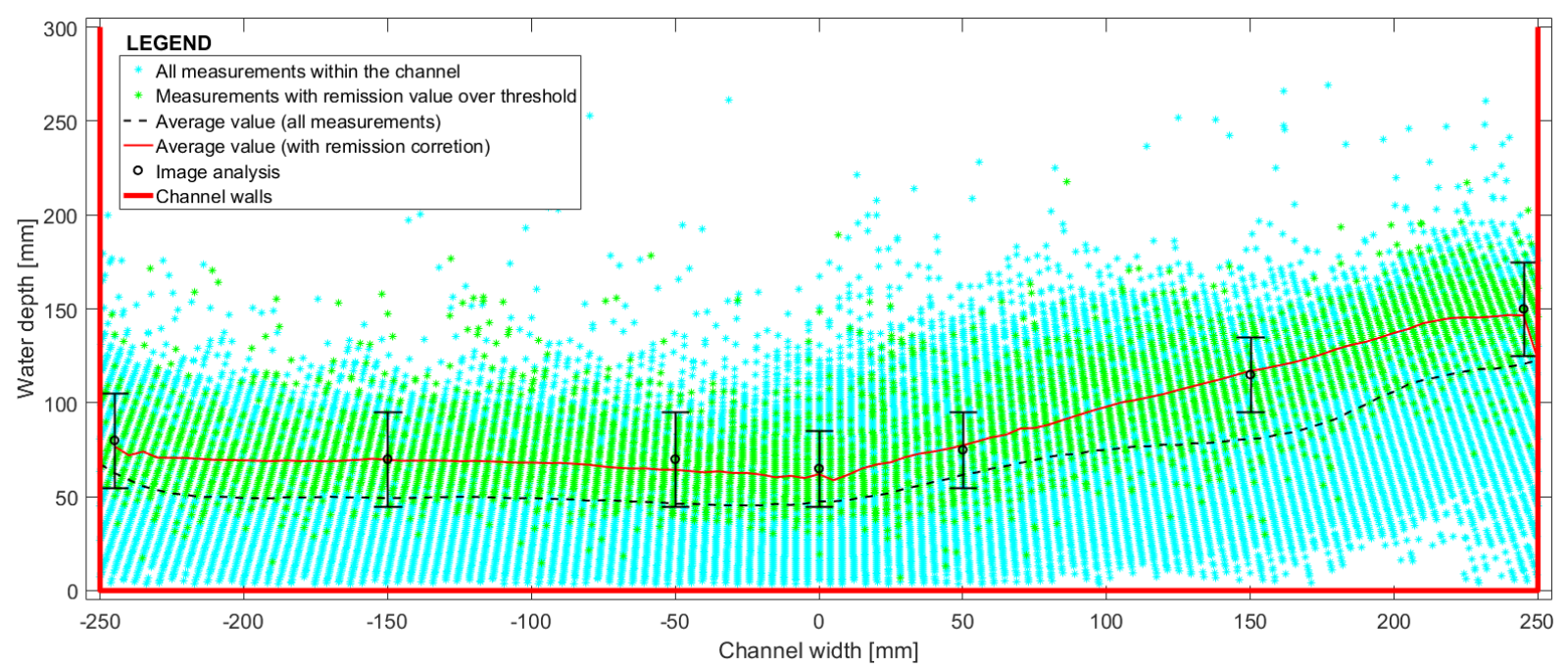

Fig. 6. The entirety of the point cloud within the channel, deviation of the filtered data with remission values above a threshold, the water surface profile, and the reference values determined through analysis of the images recorded with a high-speed camera (control section 2 on Fig. 5; scenario $h_{1}=h_{2}=20 \mathrm{~mm}, \mathrm{Fr}_{1}=8.4$ and $\mathrm{Fr}_{2}=6$ )

Table 1. Comparison of water surface measurements in selected points at control cross-section 2 using the laser scanner and image analysis with a high-speed camera

\begin{tabular}{lccccccc}
\hline & \multicolumn{9}{c}{ Measured water depth values } \\
\cline { 2 - 8 } & point 1 & point 2 & point 3 & point 4 & point 5 & point 6 & point 7 \\
\hline Image analysis using a high-speed camera [mm] & 80 & 70 & 70 & 65 & 75 & 115 & 150 \\
\hline LMS400 without filtering [mm] & 63 & 50 & 46 & 48 & 62 & 81 & 121 \\
\hline LMS400 with filtering [mm] & 77 & 70 & 65 & 62 & 77 & 117 & 147 \\
\hline Difference LMS400 and high-speed camera, in \% & -3.7 & 0.0 & -7.2 & -4.7 & +2.6 & +1.7 & -0.2 \\
\hline
\end{tabular}


points with remission values over threshold were used to construct the topography models.

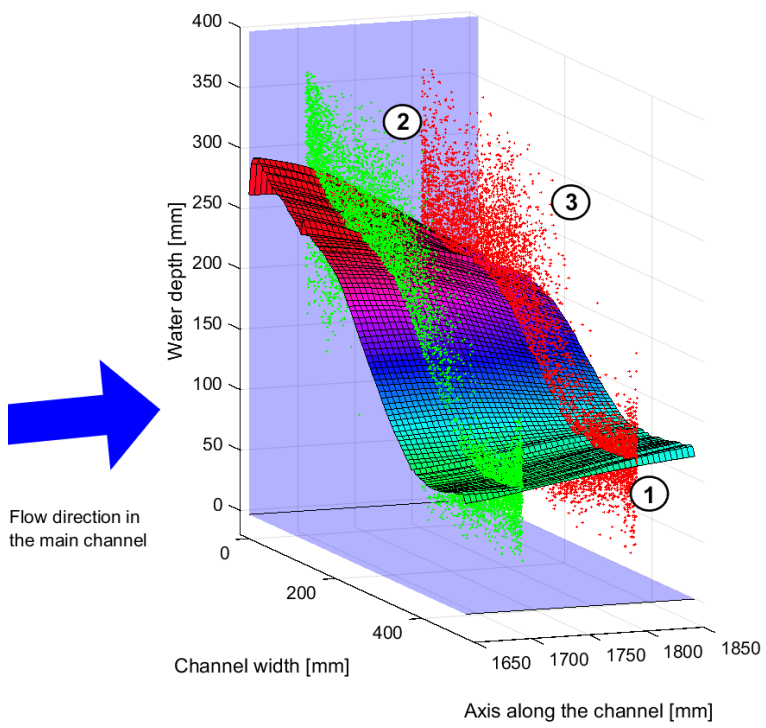

Fig. 7. Point clouds of two consecutive cross-sections and a section of the standing wave mean free water surface, constructed with post-processing of the laser scanning measurements (scenario $h_{1}=h_{2}=20 \mathrm{~mm}, \mathrm{Fr}_{1}=8.4$ and $\mathrm{Fr}_{2}=6$ )

The free water surface dynamics is shown in Fig. 7, which also shows vertical distributions of the measured reflections in two neighbouring crosssections showing the range of fluctuations around a mesh model of the surface area structured based on calculated mean values of the water surface. The Fig. 7 showing fluctuations, as evident from the entire point cloud in the individual cross-sections, reveals that the highest water surface fluctuations occur along the channel walls (points 1 and 2) where side supercritical inflow causes a high transversal dynamic of the flow and at the very top of the standing wave ridge inside the channel (point 3 ).

The design of the 3D mesh model of the entire area concerned allows for illustration of a dynamic water surface topography at a confluence and thus further processing and analysis of standing wave formations (e.g. location and size of peaks) as a function of the input parameters of the inflows. The photo above (Fig. 8a) shows the 3D topography for the case of two incoming flows with the same depth in both branches $(20 \mathrm{~mm})$, but with different Froude numbers, i.e. $F r=8.5$ at the main channel and $F r=5.6$ at the side channel. As the laser imaging progressed from top down (i.e. plan view), the measured water surface cannot show air pockets or tunnels of air occurring in the body of the wave, below the cover ridge of the wave along the circulation zone of both inflows (Fig. 8b). As seen from the photo, with high velocities of both inflows a barrel-like water flow formation occurs, i.e. a barrel roll; the barrel's inside surfaces, of course, cannot be detected from the existing scanning axes. The number of successful measurements is also low where water is not or is hardly aerated (front-left part of the Fig. 8b). In this area there are no bubbles, but water surface is slightly waved due to the water spraying. In this area we got some successful measurements with reflection at perpendicular incident on the water surface. Density of points are smaller as well as the quality, but it was still possible to generate the $3 \mathrm{D}$ mesh model. Tests show that, given the momentum of incoming flows, a single barrel can be formed in the direction of the stronger flow, or a double barrel in the case of equal flows.

Fig. 9 shows 4 topography models for the scenarios with different flow characteristics. LIDAR measurements and the topography models constructed
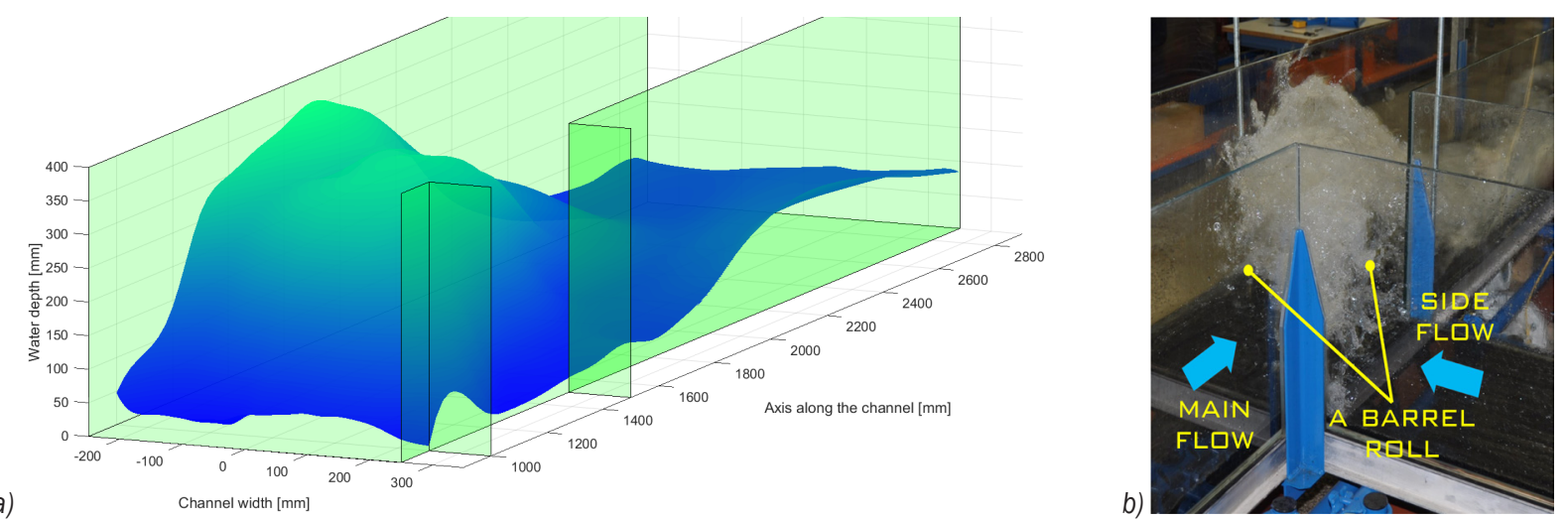

Fig. 8. a) A case of constructed water surface topography along the confluence, and b) a photo - both showing wave peaks along the wall and in the channel 

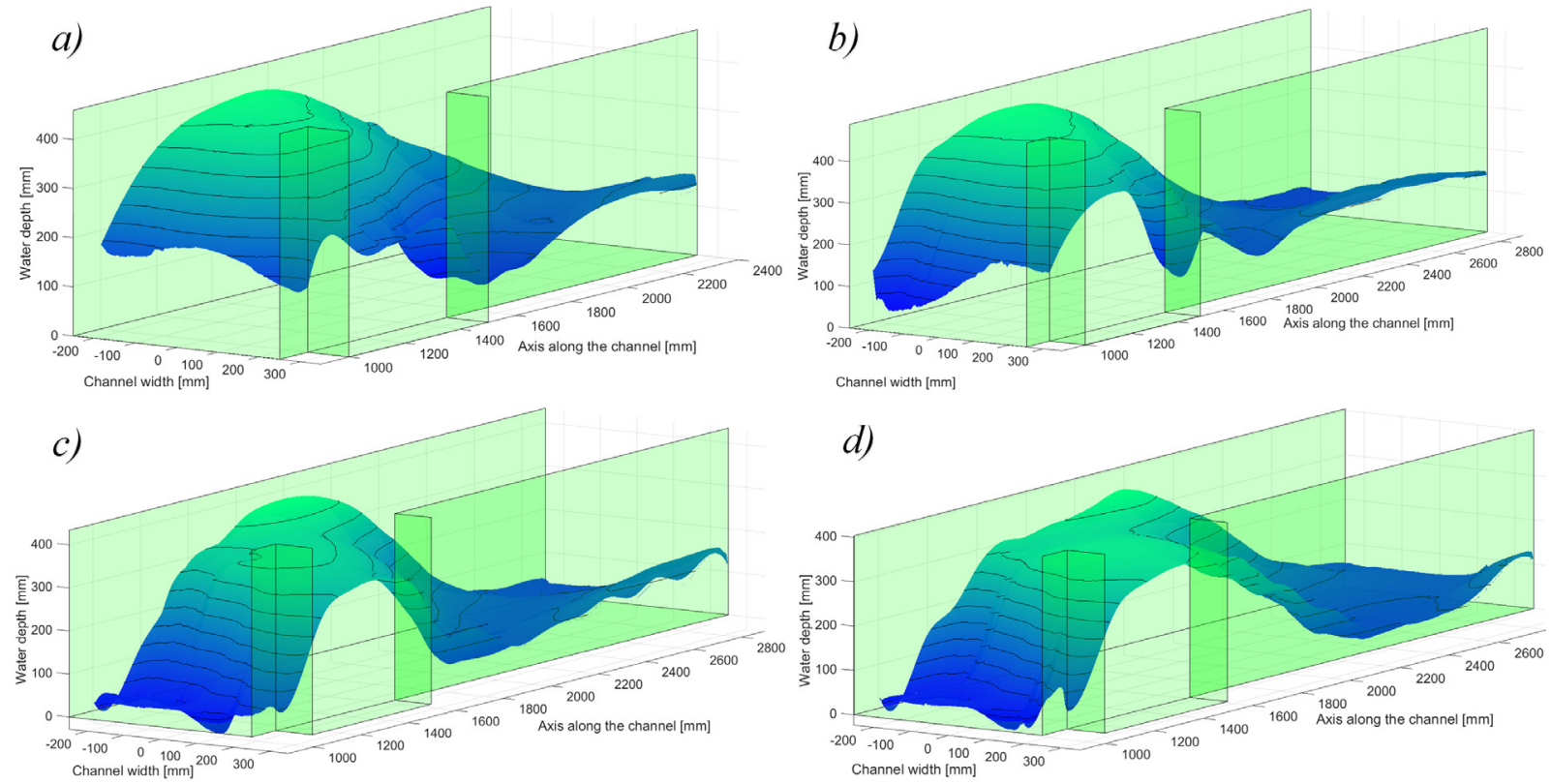

Fig. 9. 3D mesh models of water surface topography at the confluence for 4 scenarios with different flow geometry and hydraulic conditions: a) $h_{1}=h 2=25 \mathrm{~mm}, \mathrm{Fr}_{1}=7.92$ and $\left.F r_{2}=5.79 ; b\right) h_{1}=30 \mathrm{~mm}, h_{2}=20 \mathrm{~mm}, \mathrm{Fr}_{1}=6.76$ and $\mathrm{Fr}_{2}=7.04$;

c) $h_{1}=30 \mathrm{~mm}, h_{2}=10 \mathrm{~mm}, \mathrm{Fr}_{1}=5.99$ and $\mathrm{Fr}_{2}=11.99 ;$ d) $h_{1}=30 \mathrm{~mm}, h_{2}=10 \mathrm{~mm}, \mathrm{Fr}_{1}=6.9$ and $\left.\mathrm{Fr}_{2}=11.05\right)$

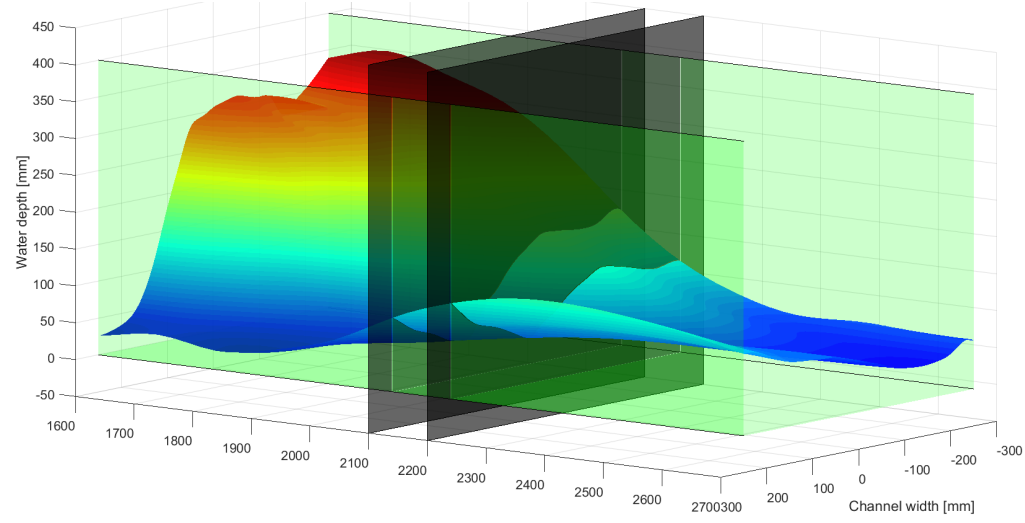

a) Axis along the channel [mm]

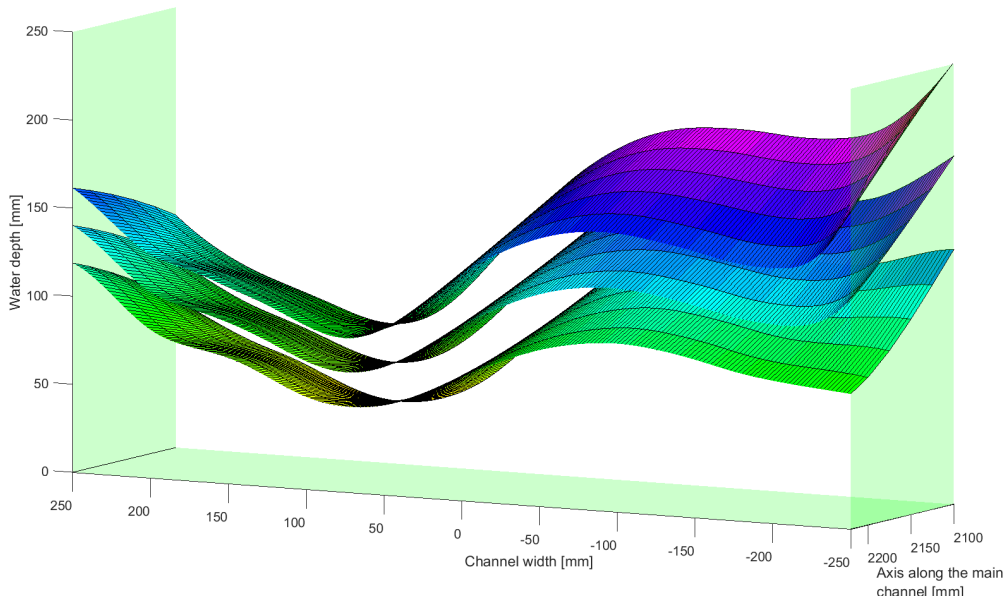

Fig. 10. a) Location indication of a standing wave section in a length of $100 \mathrm{~mm}$, and b) section of standing wave with a plot of the average free water surface and water surface envelopes by taking into account the measured fluctuations 
from them can be used to determine and visualize the structures of water flow, wave peaks and their location, points of water surface inflections, extend of standing waves etc.

Considering the (filtered) fluctuations in the individual cross-sections, it is also possible to determine the maximum and minimum water surface fluctuation envelope (e.g. without drops above the water surface). However, due to the lower number of successful measurements in the individual scans of the measured sections and particularly because the measurements were taken in the individual sections, a continuous, simultaneous illustration of temporal dynamics of water mass movement across the entire area is not possible. Nevertheless, based on the results of measuring permanent inflow from both channels we can analyse the extent to which water surface fluctuations occur also in critical locations. With knowledge of free-water surface fluctuations of these phenomena we can select appropriate solutions, when designing the hydraulic structures in practice. Fig. 10a shows a 100-mm long section (left), and Fig. 10b 3D mesh model of mean water surface as well as both measured envelopes of water surface fluctuations.

The high frequency of laser sampling allows for a highly illustrative and simultaneous representation of water level fluctuations across the entire channel cross-section. To represent the simultaneous changing of the surface in the measured section, simultaneous measurements in a mesh of points, not only in crosssections, and additional analysis of a raw data are required.

\section{CONCLUSIONS}

The undeniably wide applicability of laser scanning is particularly evident in solid state measurements if, given the complexity of surface morphology, an adequate number of observation points is selected. In their tests - but with the addition of particulate matter into the water to improve reflectivity - some authors confirmed the applicability of the laser method also when measuring the travel and transformation of waves, but mostly with a slower dynamic than in our case. An added value of this measuring method has been created by the results of this study, which testify to the method's efficiency in model studies when clean water is used, both for standing water surface measurements and for acquisition of the water surface profile with a high time variability, whose dynamics cannot be captured by conventional measurements. Notably, this method gives very good results in the case of distinctively two-phase flows. In the case of water bodies with a relatively small content of air bubbles, or small density of two-phase flow in the upper layer, this measurement method is much less appropriate, water depths are underestimated, while a precise water surface formation is difficult to determine. Hydraulic phenomena with strongly aerated upper layers of water bodies with a continuous and homogenous layer of bubbles allow for a noncontact but very precise acquisition of transversal water surface formation and thus topography of water surfaces of complex cases with aerated flow and high vertical and horizontal dynamics. Data acquisition with high temporal and spatial resolution is, particularly in the cases with strongly diversified water surface in the transversal direction, important also for calibration and verification of numerical models, as nowadays even full 3D numerical models are unable to precisely simulate flow conditions at T-junctions, i.e. in areas with a simple enough channel geometry. The construction of water surface topography from the raw point cloud of measurements using laser scanning allows for determination of waving characteristics, a further analysis of flow conditions, and the determination of the main flow structures at the confluence and its characteristics as a function of integral parameters in both inflows, thus providing the background for determining the T-junction topology. With further assumptions and additional analysis of a raw reflection point cloud, it will be possible to analyse and evaluate practically simultaneous water surface fluctuations across the whole measured cross-sections, as the laser beam movement dynamics is considerably higher than the water surface changing dynamics.

\section{REFERENCES}

[1] Webber, N.B., Greated, C.A. (1966). An investigation of flow behavior at the junction of rectangular channels. Proceedings of the Institution of Civil Engineers, vol. 34, no. 3, p. 321-334, DOI:10.1680/iicep.1966.8925.

[2] Biron, P.M., Ramamurthy, A.S., Han, S. (2004). Threedimensional numerical modeling of mixing at river confluence. Journal of Hydraulic Engineering, vol. 130, no. 3, p. 243-253, D0I:10.1061/(ASCE)0733-9429(2004)130:3(243).

[3] Pinto Coelho, M.M.L. (2015). Exprimental determination of free surface levels at open-channel junction. Journal of Hydraulic Research, vol. 53, no. 3, p. 394-399, D0l:10.1080/ 00221686.2015.1013513.

[4] Biron, P.M., Richer, A., Kirkbride, A.D., Roy, A.G., Han, S. (2002). Spatial patterns of watter surface topography at a river confluence. Earth Surface Process and Landforms, vol. 27, no. 9, p. 913-928, Dol:10.1002/esp.359. 
[5] Mignot, E., Riviere, N., Perkins, R., Paquier, A. (2008). Flow patterns in a four-branch junction with supercritical flow. Journal of Hydraulic Engineering, vol. 134, no. 6, p. 701-713. DOI:10.1061/(ASCE)0733-9429(2008)134:6(701).

[6] Schwalt, M., Hager, W.H. (1995). Experiments to supercritical junction flow. Experiments in Fluids, vol. 18, no. 6, p. 429-437, DOI:10.1007/BF00208465.

[7] Bric, V., Berk, S., Triglav Čekada, M. (2013). Quality assurance of georeferencing airborne laser scanning data for water resource management. Geodetski vestnik (Journal of the Association of Surveyors of Slovenia), vol. 57, no. 2, p. 257285, D0l:10.15292/geodetski-vestnik.2013.02.271-285. (in Slovene)

[8] Mongus, D., Triglav Čekada, M., Žalik, B. (2013). The analysis is of an automatic method for digital terrain model generation from lidar data on Slovenian test cases. Geodetski vestnik (Journal of the Association of Surveyors of Slovenia), vol. 57, no. 2, p. 245-259, DOl:10.15292/geodetskivestnik.2013.02.045-259. (in Slovene)

[9] Rak, G., Steinman, F., Gosar, L. (2008). Analysis of hydraulic properties of watercourses using GIS tools (Analiza hidravličnih lastnosti vodotokov z uporabo GIS orodja). GISs in Slovenia 2005-2006 (Geografski informacijski sistemi v Sloveniji 20052006), p. 123-131. (in Slovene)
[10] Allis, M.J., Peirson, W.L., Banner, M.L. (2011). Application of LIDAR as a measurement tool for waves. Proceedings of the

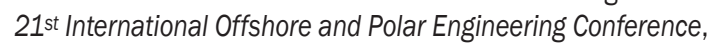
p. 19-24.

[11] Blenkingsopp, C.E., Turner, I.L., Allis M.J., Peirson, W.L., Garden, L.E. (2012). Application of LiDAR technology for measurement of time-varying free-surface profiles in a laboratory wave flume. Coastal Engineering, vol. 68, p. 1-5, D0l:10.1016/j.coastaleng.2012.04.006.

[12] Streicher, M., Hofland, B., Lindenbergh, R.C. (2013). Laser ranging for monitoring water waves in the new Deltares Delta Flume. ISPRS Annals of the Photogrammetry, Remote Sensing and Spatial Information Sciences, vol. 2, no. 5, p. 271-276, DOl:10.5194/isprsannals-II-5-W2-271-2013.

[13] Hofland, B., Diamantidou, E., van Steeg, P., Meys, P. (2015). Wave runup and wave overtopping measurements using a laser scanner. Coastal Engineering, vol 106, p. 20-29, D0l:10.1016/j.coastaleng.2015.09.003.

[14] Rak, G., Hočevar, M., Steinman, F. (2017). Measuring water surface topography using laser scanning. Flow Measurements and Instrumentation, vol. 56, p. 35-44, D0l:10.1016/j. flowmeasinst.2017.07.004. 